Dated: 17 August 2018

\title{
Improved constraint on the primordial gravitational-wave density using recent cosmological data and its impact on cosmic string models
}

\begin{abstract}
The production of a primordial stochastic gravitational-wave background by processes occuring in the early Universe is expected in a broad range of models. Observing this background would open a unique window onto the Universe's evolutionary history. Probes like the Cosmic Microwave Background (CMB) or the Baryon Acoustic Oscillations (BAO) can be used to set upper limits on the stochastic gravitational-wave background energy density $\Omega_{\mathrm{GW}}$ for frequencies above $10^{-15} \mathrm{~Hz}$. We perform a profile likelihood analysis of the Planck CMB temperature anisotropies and gravitational lensing data combined with WMAP low- $\ell$ polarization, BAO, South Pole Telescope and Atacama Cosmology Telescope data. We find that $\Omega_{\mathrm{GW}} \mathrm{h}_{0}^{2}<3.8 \times 10^{-6}$ at $95 \%$ confidence level for adiabatic initial conditions which improves over the previous limit by a factor 2.3. Assuming that the primordial gravitational waves have been produced by a network of cosmic strings, we have derived exclusion limits in the cosmic string parameter space. If the size of the loops is determined by gravitational back-reaction, string tension values greater than $\sim 4 \times 10^{-9}$ are excluded for a reconnection probability of $10^{-3}$.
\end{abstract}

Sophie Henrot-Versillé ${ }^{1}$, Florent Robinet ${ }^{1}$, Nicolas Leroy ${ }^{1}$, Stéphane Plaszczynski ${ }^{1}$, Nicolas Arnaud ${ }^{1}$, Marie-Anne Bizouard $^{1}$, Fabien Cavalier ${ }^{1}$, Nelson Christensen ${ }^{1,2}$, François Couchot $^{1}$, Samuel Franco ${ }^{1}$, Patrice Hello ${ }^{1}$, Dominique Huet ${ }^{1}$, Marie Kasprzack ${ }^{1}$, Olivier Perdereau ${ }^{1}$, Marta Spinelli ${ }^{1}$, Matthieu Tristram ${ }^{1}$

${ }^{1}$ LAL, Univ Paris-Sud, CNRS/IN2P3, Orsay, France

${ }^{2}$ Physics and Astronomy, Carleton College, Northfield, MN 55057, USA 


\section{Introduction}

A stochastic background is expected to form from the incoherent superposition of a large number of gravitational wave (GW) signals emitted by many sources of astrophysical and cosmological origin. Examples of GW astrophysical sources are compact binary coalescence, core collapse supernovae or rotating neutron stars [1, 2, 3]. Among the cosmological sources, many GW generation mechanisms have been proposed covering a broad range of frequencies, such as the amplification of quantum vacuum fluctuations during inflation [4, 5, 6, 7, 8, first order phase transitions [9, 10, cosmic strings [11, 12, 13] and pre Big-Bang models [14, 15]. These GWs provide a unique probe of the evolution of the Universe from its birth as they travel through space-time with virtually no interaction with matter.

The stochastic background of GWs is described in terms of its energy spectrum as function of the frequency:

$$
\Omega_{\mathrm{GW}}(f)=d \Omega_{\mathrm{GW}} / d(\ln f),
$$

where $\Omega_{\mathrm{GW}}$ is the total energy density of GWs relative to the critical energy density. This spectrum is constrained for specific frequency ranges by direct GW searches. Assuming a flat spectrum, the LIGO and Virgo collaborations recently set the limit $\Omega_{\mathrm{GW}}(f \simeq 100 \mathrm{~Hz}) \times\left(\mathrm{h}_{0} / 0.68\right)^{2}<5.6 \times 10^{-6}\left[16\right.$, where $\mathrm{h}_{0}=H_{0} /\left(100 \mathrm{~km} \mathrm{~s}^{-1} \mathrm{Mpc}^{-1}\right)$ is the reduced Hubble parameter. Pulsar timing experiments provide a constraint at much lower frequencies: $\Omega_{\mathrm{GW}}(f=2.8 \mathrm{nHz}) \times\left(\mathrm{h}_{0} / 0.73\right)^{2}<1.3 \times 10^{-9}$ [17]. These constraints apply to any GW background, both of cosmological and astrophysical origin.

As far as cosmological gravitational waves (CGW) are concerned, they could ultimately be detected, if generated by inflation models, through the measurement of the tensor-to-scalar ratio with low- $\ell$ Cosmic Microwave Background (CMB) B polarization anisotropies [18, 19]. Still indirect bounds on their energy density can be set from different cosmological probes. For instance, Big Bang Nucleosynthesis (BBN), through the measurement of the light element abundances gives $\Omega_{\mathrm{GW}} \mathrm{h}_{0}^{2}<8.1 \times 10^{-6}$ for all frequencies above $10^{-10} \mathrm{~Hz}[20,21,22]$. CMB and Baryon Acoustic Oscillation (BAO) data can also be used to set limits on a CGW background energy density for frequencies greater than $10^{-15} \mathrm{~Hz}[23,24]$.

In this article, we have revisited the constraints on $\Omega_{\mathrm{GW}} \mathrm{h}_{0}^{2}$ using the high-precision measurements of the CMB properties from the Planck collaboration [25, 26] combined with up-to-date BAO data [27]. The study is made within the $\Lambda_{\mathrm{CDM}}$ scenario which has proved to be able to successfully describe a wide range of cosmological data [25]. In order to be as model independent as possible, we have considered the case for which the CGWs are produced under adiabatic conditions. Within this framework, the influence of CGWs on the CMB power spectrum is expected to be the same as extra massless neutrino species [23, 24]. We then perform a profile likelihood analysis on the effective number of relativistic degrees of freedom, $\mathrm{N}_{\text {eff }}$, with different hypotheses on the sum of neutrinos masses $\left(\sum m_{\nu}\right)$, and derive the corresponding upper limits on $\Omega_{\mathrm{GW}} \mathrm{h}_{0}^{2}$. The limit derived with $\sum m_{\nu}$ free in the fit is thereafter used to constrain cosmic string 
model parameters assuming the CGW background was produced by a network of cosmic strings.

In section 2, we briefly present the theoretical framework used to constrain the CGW background energy density. The description of the cosmological data, the

statistical procedure and the results on $\Omega_{\mathrm{GW}} \mathrm{h}_{0}^{2}$ are discussed in Section 3 . The impact on cosmic string models is described in Section 4. Finally we discuss in Section 5 the future prospects. Throughout this paper, we take the speed of light and the reduced Planck constant equal to 1 .

\section{The stochastic gravitational-wave background density}

The radiation energy density relative to the critical density, $\Omega_{\text {rad }}$, can be written as the sum of the relativistic contributions of photons $(\gamma)$, neutrinos $(\nu)$, and any possible extra radiation $(x)$ :

$$
\Omega_{\text {rad }}=\Omega_{\gamma}+\Omega_{\nu}+\Omega_{x} .
$$

Introducing the effective number of relativistic degrees of freedom, $\mathrm{N}_{\text {eff }}$, this expression can be re-written as follows [21, 28]:

$$
\Omega_{\text {rad }}=\left[1+\frac{7}{8} \mathrm{~N}_{\mathrm{eff}}\left(\frac{4}{11}\right)^{4 / 3}\right] \Omega_{\gamma} .
$$

Under the assumption that only photons and standard light neutrinos contribute to the radiation energy density, $\mathrm{N}_{\text {eff }}$ is equal to the effective number of neutrinos and is constrained by the measurement of the decay width of the $\mathrm{Z}$ boson [29]. Taking into account the residual heating of the neutrino fluid due to electron-positron annihilation, its predicted value is $\mathrm{N}_{\mathrm{eff}} \simeq 3.046$ [28]. Any deviation from this value can be attributed to extra relativistic radiation, including massless sterile neutrino species, axions [30, 31, decay of non-relativistic matter [32], GWs [23], extra dimensions [33, 34, 35], early dark energy [36], asymmetric dark matter [37], or leptonic asymmetry [38]. Assuming that a stochastic CGW background contributes to this extra relativistic radiation energy density, one can set an upper limit on the CGW background energy density, $\Omega_{\mathrm{GW}}$, using Eq. 35:

$$
\Omega_{\mathrm{GW}} \leq \frac{7}{8}\left(\frac{4}{11}\right)^{4 / 3}\left(\mathrm{~N}_{\mathrm{eff}}-3.046\right) \Omega_{\gamma} .
$$

Using $T=2.7255 \pm 0.0006 \mathrm{~K}$ for the photon temperature today [39], one can deduce, from [29], the numerical value:

$$
\Omega_{\gamma}=2.47310^{-5} / h_{0}^{2} .
$$

Eq. 4 then writes:

$$
\Omega_{\mathrm{GW}} \mathrm{h}_{0}^{2} \leq 5.610^{-6}\left(\mathrm{~N}_{\mathrm{eff}}-3.046\right) .
$$

In the next section, we will measure $\mathrm{N}_{\text {eff }}$ using the most recent cosmological data and Eq. 6 will be used to constrain the CGW background energy density. 


\section{New constraints from Planck}

\subsection{Data, models and statistical strategy}

$\mathrm{CMB}$ and $\mathrm{BAO}$ data have been used in the following analysis. The CMB data are composed of the temperature anisotropy likelihood [25] (hereafter called Planck), and the CMB lensing from Planck [26] (Lensing), WMAP low- $\ell$ polarization (WP), SPT and ACT [40, 41] high- $\ell$ spectra (HighL) data. We combine them with BOSS BAO DR9 [42, 43, 44] data (BAO). In addition, the robustness of the results has been checked against BOSS BAO DR11 [27] data.

Profile likelihoods $\mathcal{L}$ of the $\mathrm{N}_{\text {eff }}$ parameter have been built; they are obtained by fixing $\mathrm{N}_{\text {eff }}$ to predefined values, and maximizing the likelihood function over all the other dimensions of the parameters space. The combined likelihood includes a total of 37 cosmological and nuisance parameters as explained in [45. The maximisation is done using the Minuit software [46], interfaced to the CLASS Boltzmann solver [47, which, from a set of input cosmological parameters, computes the corresponding CMB temperature and polarization anisotropy power spectra. The tuning of the CLASS precision parameters has been done according to [45]. Finally, a limit on $\Omega_{\mathrm{GW}} \mathrm{h}_{0}^{2}$ using Eq. (6) is computed using the Feldman-Cousins prescription [48] to take into account the condition $\Omega_{\mathrm{GW}} \mathrm{h}_{0}^{2} \geq 0$.

Since the $\Omega_{\mathrm{GW}} \mathrm{h}_{0}^{2}$ limit is deduced from the $\mathrm{N}_{\mathrm{eff}}$ measurement, several neutrino models have been investigated for, in order to study the sensitivity of our results to this unknown. Three cases have been considered as (extended-) $\Lambda_{\mathrm{CDM}}$ scenarios: the sum of the neutrino masses $\left(\sum m_{\nu}\right)$ has either been set to 0 , or assumed to be $0.06 \mathrm{eV}$ as in [49, 45] which is consistent with [50], or assumed to be free to vary and fitted for for in the statistical procedure.

\subsection{Results}

The measured 95\% CL upper limits are summarized in Table 1. We have checked that the underlying $\mathrm{N}_{\text {eff }}$ measurement is fully compatible with [49]. For instance, for the Planck $+\mathrm{WP}+$ HighL $+\mathrm{BAO}$ dataset and assuming $\sum m_{\nu}=0.06 \mathrm{eV}$, the profile likelihood method gives $\mathrm{N}_{\mathrm{eff}}=3.29 \pm 0.54$ at a $95 \% \mathrm{CL}$, very close to the value $3.30_{-0.51}^{+0.54}$ of [49]. This shows that the results from both analyses using different Bolzman solvers and different statistical methods are equivalent.

Fig. 1 shows the $-2 \log \mathcal{L}$ functions for the full dataset, for the three hypotheses of the neutrino masses discussed above. The corresponding limits are reported in Table 1 . The results are very robust to the considered neutrino models, since fitting the $\sum m_{\nu}$ parameter does not significantly change the upper limits. In addition, the use of the DR11 BAO data in place of the DR9 does not improve the limit. On the other hand, CMB lensing data are very useful for improving the constraints as the upper limit gets tighter by almost $20 \%$.

In the following, we consider the limit obtained with the full dataset and with $\sum m_{\nu}$ 


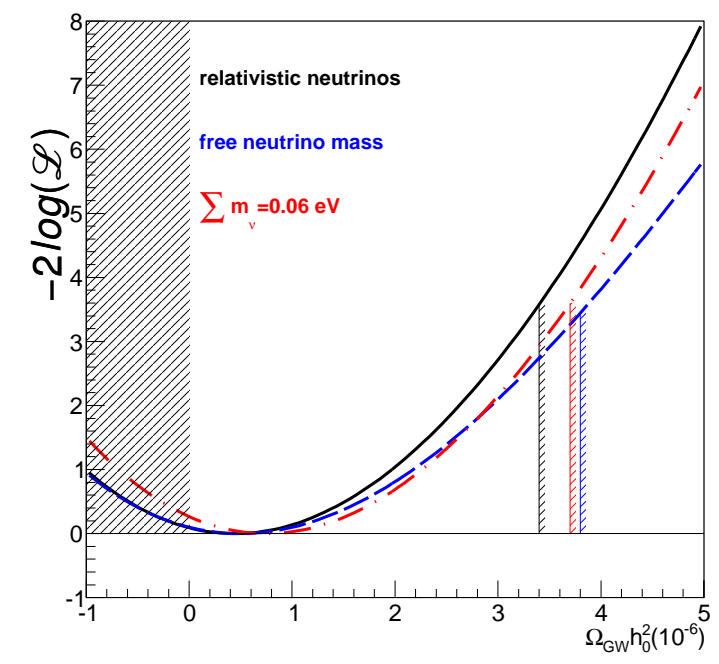

Figure 1. Profile likelihoods of $\Omega_{\mathrm{GW}} \mathrm{h}_{0}^{2}$ with the full Planck+WP+HighL+BAO+Lensing dataset (see Section 3.1 for details) in three neutrino models: when the neutrinos are considered to be massless (in black full line), when the sum of the neutrino masses is assumed to be $\sum m_{\nu}=0.06 \mathrm{eV}$ (in red dashed and dotted line), and when the neutrino mass is let free to vary in the fit (in blue dahed line). The grey area correspond to the unphysical $\Omega_{\mathrm{GW}} \mathrm{h}_{0}^{2} \leq 0$ region. The limits are then derived using the Feldman-Cousins prescription and shown as vertical lines.

\begin{tabular}{|l|c|c|}
\hline data & model & $95 \%$ CL Limits \\
\hline Planck+WP+HighL+BAO & $\sum m_{\nu}=0$ & $<4.1 \times 10^{-6}$ \\
Planck+WP+HighL+BAO +Lensing & & $<3.4 \times 10^{-6}$ \\
\hline Planck+WP+HighL+BAO & $\sum m_{\nu}=0.06 \mathrm{eV}$ & $<3.7 \times 10^{-6}$ \\
Planck+WP+HighL+BAO+Lensing & & $<3.7 \times 10^{-6}$ \\
Planck+WP+HighL +BAODR11+Lensing & & $<4.1 \times 10^{-6}$ \\
\hline Planck+WP+HighL +BAO & $\sum m_{\nu}$ free & $<\mathbf{3 . 8} \times \mathbf{1 0}^{-\mathbf{6}}$ \\
Planck+WP+HighL +BAO+Lensing & & \\
\hline
\end{tabular}

Table 1. 95\% CL limits on $\Omega_{\mathrm{GW}} \mathrm{h}_{0}^{2}$ for different datasets and different neutrino mass hypothesis $\left(\sum m_{\nu}\right.$ free means that the sum of the neutrino masses is a free parameter in the profile likelihood determination). Those limits are valid for frequencies above $10^{-15} \mathrm{~Hz}$. The bold value is the one used in the following sections.

being a free parameter,

$$
\Omega_{\mathrm{GW}} \mathrm{h}_{0}^{2}<3.8 \times 10^{-6}
$$

These upper limits can be compared to the ones previously derived in [23] and [24]. In the analysis of [23], the quoted limit for adiabatic initial condition is $4 \times 10^{-5}$ at 95\% CL. In their analysis, they assumed that the contribution of the standard neutrino only-component of $\mathrm{N}_{\mathrm{eff}}$ is 3.04 and the neutrino masses were free to vary. This result is directly comparable to our limit when fitting for $\sum m_{\nu}$, and is an order of magnitude 
larger. A more recent result [24] gave $\Omega_{\mathrm{GW}} \mathrm{h}_{0}^{2}<8.7 \times 10^{-6}$ at a 95\% CL assuming massless neutrino species as is done for the results presented in the first lines of Table 1 . leading to a result more than a factor two above our final results. They also quote a limit of $10^{-6}$ for homogeneous initial conditions which are not investigated here. Apart from the fact that the limit obtained in this paper is the lowest as of today for adiabatic initial conditions, we have also shown that it is robust to the assumptions made on the neutrino mass.

\section{Constraints on cosmic strings models}

The limit on the primordial stochastic GW background can be used to constrain cosmic string models, for if cosmic strings exist, they would be a powerful source of GWs. Bursts of GWs are emitted by string features called cusps and kinks that propagate along string loops, a result of cosmic string interactions. The GWs emitted by cosmic strings are described by the dimensionless string tension parameter $G \mu$, where $G$ is the Newton constant and $\mu$ the mass per unit length of the string. Recent results from Planck [51] showed that, in order to be compatible with the measured CMB anisotropies, the string tension had to be lower than $1.5 \times 10^{-7}$ and $3.2 \times 10^{-7}$ for Nambu-Goto and AbelianHiggs strings respectively. When two string segments meet, they have a probability to exchange ends; strings are said to intercommute or to reconnect. A network of cosmic strings is then also characterized by a reconnection probability, $p$. For topological strings, $p$ is essentially 1 while it can be much smaller for cosmic strings formed in the context of string theory (superstrings) [52]. When a single string reconnects with itself, a closed loop breaks off, oscillates and radiates gravitationally through the formation of cusps and kinks. This mechanism was first described in [53] and has been extended more recently in [13]. For clarity, the main analysis steps of [13], leading to constraints on cosmic string models, are summarized below.

When considering an incoherent superposition of GWs emitted by cusps or kinks, the GW spectrum, defined in Section 1, is given by

$$
\Omega_{\mathrm{GW}}(f)=\frac{4 \pi^{2}}{3 H_{0}^{2}} f^{3} \int d z \int d l h^{2}(f, z, l) \frac{d^{2} R(z, l)}{d z d l} .
$$

The GW strain amplitude produced by a cusp or a kink at a redshift $z$ is represented by $h$. The GW burst rate per loop length per redshift is $\frac{d^{2} R(z, l)}{d z d l}$. The loop size is typically taken as a fraction of the horizon size at cosmic time $t: l \sim \alpha t$. Early simulations of cosmic string evolution, such as [54], suggested that the loop size was determined by the gravitational back-reaction, in which case loops are short-lived and $\alpha \leq \Gamma G \mu$, where $\Gamma \sim 50$ is related to the fraction of power going into GWs. Recent simulations [55, 56] showed that the gravitational back-reaction scale is irrelevant and the formation of large loops is favored $(\alpha \sim 0.05)$. The small and large loop scenarios strongly impact the loop density that is used to compute the expected GW burst rate. In the following we examine both cases and derive the corresponding upper limits using the constraint obtained in Section 3 . 
Improved constraint on the primordial gravitational-wave density using recent cosmological data and its impa

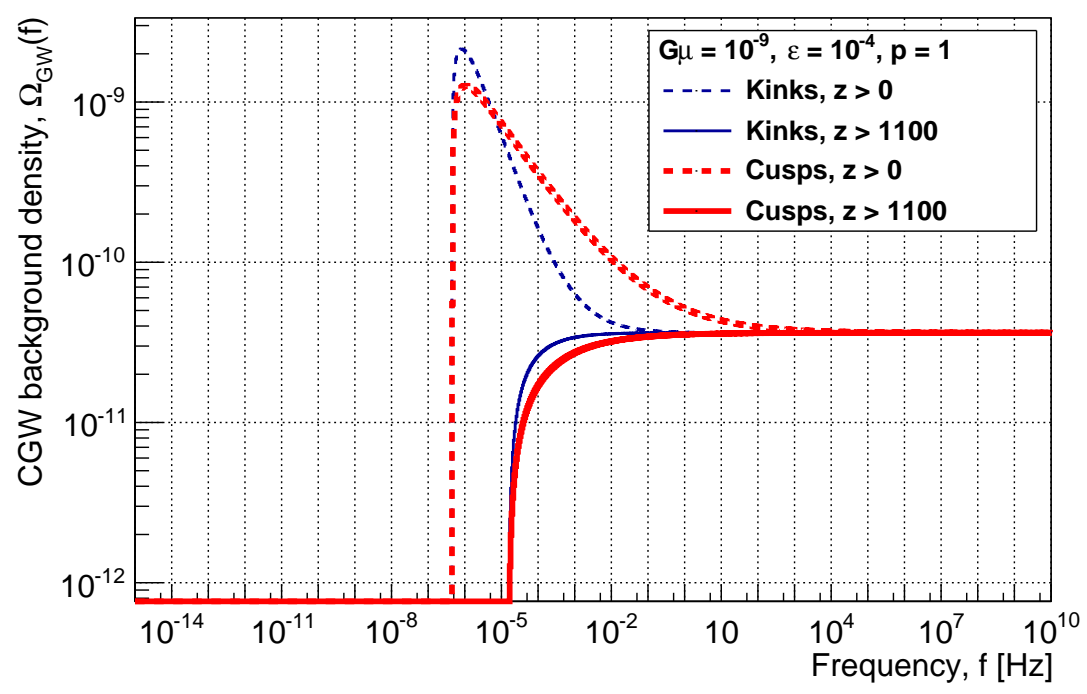

Figure 2. Kink (thin blue lines) and cusp (thick red lines) predicted spectra for small cosmic string loops $(\alpha \ll \Gamma G \mu)$ using $G \mu=10^{-7}, \varepsilon=10^{-4}$ and $p=1$. These spectra are derived numerically from Eq. 10 and Eq. 11 using $z>1100$ for GWs produced prior to the photon decoupling and $z>0$ for GWs produced up to today.

If loop sizes at the time of formation were driven by the gravitational back-reaction, the rate of GW bursts would be computed using a loop density

$$
n(l, t)=c(z)(p \Gamma G \mu)^{-1} t^{-3} \delta(l-\alpha t),
$$

where $c(z)=1+9 z /\left(z+z_{e q}\right)$ [53, 13] and $z_{e q}=3366$ [49] is the redshift at matterradiation equality. This expression simplifies the integral over $l$ in Eq. 8 to

$$
\Omega_{\mathrm{GW}}{ }^{c u s p}(f)=\frac{2 G \mu \pi^{2} H_{0}^{1 / 3}}{3 p \alpha^{1 / 3} \Gamma f^{1 / 3}} \times \int d z \frac{c(z) \varphi_{V}(z) \Theta\left[1-\left(f(1+z) H_{0}^{-1} \alpha \varphi_{t}(z)\right)^{-1 / 3}\right]}{(1+z)^{7 / 3} \varphi_{r}^{2}(z) \varphi_{t}^{10 / 3}(z)},
$$

for cusps and

$$
\Omega_{\mathrm{GW}}{ }^{k i n k}(f)=\frac{4 G \mu \pi^{2} H_{0}^{2 / 3}}{3 p \alpha^{2 / 3} \Gamma f^{2 / 3}} \times \int d z \frac{c(z) \varphi_{V}(z) \Theta\left[1-\left(f(1+z) H_{0}^{-1} \alpha \varphi_{t}(z)\right)^{-1 / 3}\right]}{(1+z)^{8 / 3} \varphi_{r}^{2}(z) \varphi_{t}^{11 / 3}(z)},
$$

for kinks, where we used the gravitational waveform $h$ and the rate $\frac{d^{2} R(z, l)}{d z d l}$ modeled in [13]. The expressions differ because kinks and cusps have different waveforms and different GW beaming angles. Three dimensionless functions, $\varphi_{t}(z), \varphi_{r}(z)$ and $\varphi_{V}(z)$, are used to reflect the redshift dependence of cosmic time, proper distance and volume, respectively. These cosmological functions are numerically computed using the expressions defined in [13] and the cosmological parameters from [49].

For this study, we compute the GW spectrum for kinks and cusps using a set of cosmic string parameters $(G \mu, \varepsilon, p)$, where $\varepsilon \equiv \alpha /(\Gamma G \mu)$, with $\varepsilon<1$, is another parameterization of the loop size. Examples of such spectra are presented in Fig. 2. The 

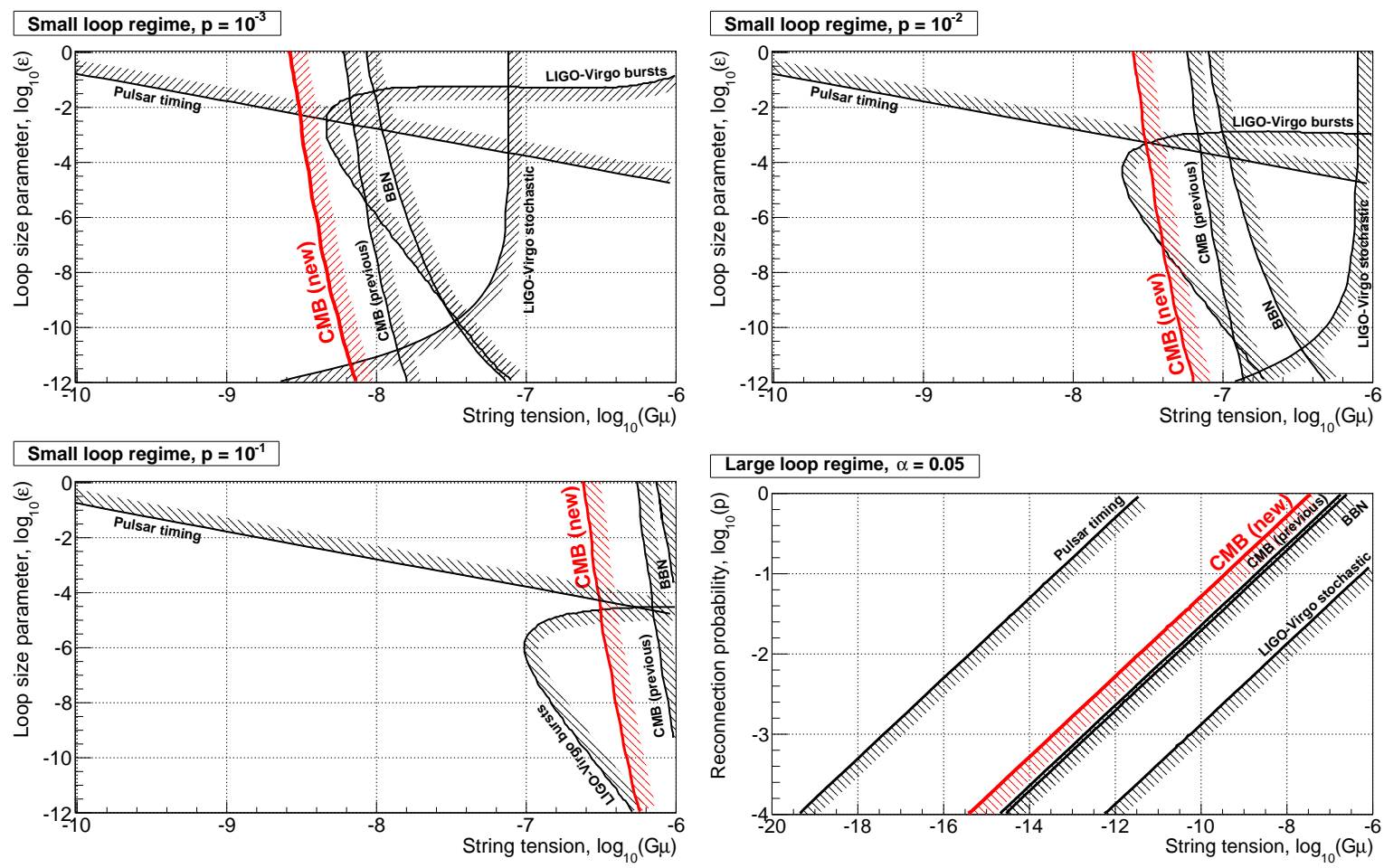

Figure 3. Constraints on cosmic string parameters $(G \mu, \varepsilon)$ assuming loops are small and fixing $p=10^{-3}$ (top-left), $p=10^{-2}$ (top-right), and $p=10^{-3}$ (bottom-left). The lower-right plot shows exclusion contours in the $(G \mu, p)$ plane when loops are large and for $\alpha=0.05$ [56]. Our new constraints, derived from $\Omega_{\mathrm{GW}} \mathrm{h}_{0}^{2}<3.8 \times 10^{-6}$, are compared to existing limits obtained from previous CMB $\left(\Omega_{\mathrm{GW}} \mathrm{h}_{0}^{2}<8.7 \times 10^{-6}[24]\right), \mathrm{BBN}\left(\Omega_{\mathrm{GW}} \mathrm{h}_{0}^{2}<8.1 \times 10^{-6}[22]\right)$, LIGO-Virgo stochastic $\left(\Omega_{\mathrm{GW}}(f \simeq 100 \mathrm{~Hz}) \times\left(\mathrm{h}_{0} / 0.68\right)^{2}<5.6 \times 10^{-6}[16]\right)$, LIGO-Virgo bursts [57] and pulsar timing $\left(\Omega_{\mathrm{GW}}(f=2.8 \mathrm{nHz}) \times\left(\mathrm{h}_{0} / 0.73\right)^{2}<1.3 \times 10^{-9}[17]\right)$ data analyses.

cosmic string parameter space is scanned and, for each parameter set, we compute the spectrum integral $\int d(\ln f)\left(\Omega_{\mathrm{GW}}{ }^{c u s p}(f)+\Omega_{\mathrm{GW}}{ }^{k i n k}(f)\right)$. The integration is performed from $10^{-15} \mathrm{~Hz}$ up to $10^{10} \mathrm{~Hz}$, to encompass GWs produced after the phase transition which produced the string network and before the time of the photon decoupling, and using $h_{0}=0.6780 \pm 0.0077$ [49]. Cosmic string parameters are excluded by requiring this integral to be smaller than the upper limit on $\Omega_{\mathrm{GW}}$ obtained in Section 3 . The upper plots and the lower-right plot of Fig. 3 present the resulting exclusion contours at $95 \% \mathrm{CL}$ (in red and labeled "CMB new") when $p$ is fixed at $10^{-3}, 10^{-2}$ and $10^{-1}$.

Fig. 3 also displays existing constraints which have been derived using the model of [13] and rescaled with a uniform set of cosmological parameters [49]. Our new constraint on the string tension is tighter by more than a factor 2 (resp. a factor 4) as compared to previous results using CMB data [24] (resp. BBN data 22]). When compared to LIGO-Virgo results from searches for a stochastic GW background [16], our new results are an improvement by about an order of magnitude. We also consider the recent search for individual GW bursts from cusps [57, 58] compared to which our constraints does not scale the same way with $p$ in the $(G \mu, \varepsilon)$ plane: our new bound is 
only competitive for $p \lesssim 10^{-2}$. Finally, we have computed the constraints from pulsar timing data using the limit on $\Omega_{\mathrm{GW}}(f)$ from [17; it complements our result for $\varepsilon \gtrsim 10^{-3}$. It is important to note that for both the pulsar timing and the LIGO-Virgo results, the constraints are not exactly comparable as these results also include GWs produced after the photon decoupling团

We also examine the case where loops are large and their size is set by the largescale dynamics of the string network, as suggested by recent simulations [55, 56]. The loop size is no longer parameterized by $\varepsilon ; \alpha$ is used instead. We use the analytical approximation derived in [13] where the CGW spectrum is flat for the radiation era $\left(z>z_{e q}\right)$. This result is obtained using a loop density, $n(l, t)$, specifically modeled for the radiation era [58] and integrated over in Eq. 8. For both cusps and kinks the CGW spectrum is approximated by

$$
\Omega_{\mathrm{GW}}(f)=\frac{192 \pi^{3} \sqrt{\alpha \Gamma G \mu}}{3 z_{e q} \Gamma^{2} p} .
$$

The lower-right plot of Fig. 3 shows how the $G \mu-p$ plane is constrained when fixing $\alpha$ at 0.05 [56]. As expected when using a $f$-independent spectrum, the best constraint is provided by the tightest limit on $\Omega_{\mathrm{GW}}(f)$, independently of the frequency band. In this context, the pulsar timing constraint is the most competitive one.

\section{Conclusions}

Assuming that the deviation of $\mathrm{N}_{\text {eff }}$ from its expected value is due to the CGW background, we have analyzed the recent CMB (from Planck, WMAP low- $\ell$ polarization, SPT and ACT [25, 26, 40, 41]) and BOSS BAO DR9 data [42, 43, 44] assuming adiabatic initial conditions. Using a profile likelihood method we have derived an upper limit on the CGW energy density for frequencies greater than $10^{-15} \mathrm{~Hz}: \Omega_{\mathrm{GW}} \mathrm{h}_{0}^{2}<3.8 \times 10^{-6}$ at $95 \%$ confidence level. With $\mathrm{h}_{0}=0.678$ [49] this implies $\Omega_{\mathrm{GW}}<8.3 \times 10^{-6}$. This result is a factor 2.3 better than previous ones [23, 24], and is robust to the neutrino mass models. We have shown that adding the lensing CMB data to the Planck temperature measurements significantly improves the constraints. On the contrary, the use of DR11 BAO data [27] does not add enough information to significantly change the upper limit.

Under the hypothesis that the primordial GWs can be attributed to a network of cosmic strings, we have computed exclusion regions in the string parameter space for any possible size of loops. The best limit is obtained in the scenario where the size of loops is dictated by the GW back-reaction. In particular, in the context of string theory, if the cosmic string reconnection probability is $10^{-3}$, we exclude models with $G \mu \gtrsim 4 \times 10^{-9}$. For the large loop case, the best limits are still provided by the pulsar timing experiments [17].

$\ddagger$ The $z$ integrations of Eq. 10 and Eq 11 are performed using $z>1100$ for CMB, $z>5.5 \times 10^{9}$ for $\mathrm{BBN}$, and $z>0$ for LIGO-Virgo stochastic and pulsar timing results. 
Our upper bound on the adiabatic energy density and the BBN limit $\left(\Omega_{\mathrm{GW}} \mathrm{h}_{0}^{2}<\right.$ $8.1 \times 10^{-6}$ [22, 59]) covers a very large range of frequencies, $10^{-15}$ and $10^{-10} \mathrm{~Hz}$ and above respectively, while the direct search limits set with LIGO-Virgo data $\left(\Omega_{\mathrm{GW}}(f \simeq 100 \mathrm{~Hz}) \times\left(\mathrm{h}_{0} / 0.68\right)^{2}<5.6 \times 10^{-6}[16]\right)$ and pulsar timing experiments $\left(\Omega_{\mathrm{GW}}(f=2.8 \mathrm{nHz}) \times\left(\mathrm{h}_{0} / 0.73\right)^{2}<1.3 \times 10^{-9}[17]\right)$ are obtained over a much smaller frequency band. The sensitivity of the next generation of interferometric GW detectors will improve by a factor 10, especially at low frequencies (down to $10 \mathrm{~Hz}$ ). With this sensitivity, the GW background energy density constraint will be of the order of $\Omega_{\mathrm{GW}}(f \simeq 10-150 \mathrm{~Hz}) \lesssim 10^{-9}$ (assuming a year of data at LIGO-Virgo design sensitivity) [16].

Future Planck measurements using polarization may slightly reduce the error on $\mathrm{N}_{\text {eff. }}$. In the coming years, ground based or balloon borne CMB observation projects should also be able to improve on this measurement [60, 61, 62, 63, 64, 65, 66]. In the long term, Euclid [67] or LSST [68] will reduce the error on $\mathrm{N}_{\text {eff }}$ down to 0.1 at $1 \sigma$, a factor 2 smaller than the current error. Still, the prediction of these sensitivities in terms of achievable upper limits on the CGW background energy density depends highly on the $\mathrm{N}_{\text {eff }}$ value itself within the hypotheses assumed in this paper. More constraints should come in ten years from now, from high precision ground-based instruments measuring the CMB polarization anisotropies. For example the forecasted stage-IV CMB polarization experiment CMB-S4 should allow one to achieve an error on $\mathrm{N}_{\text {eff }}$ of 0.02 at $68 \%$ CL [69], i.e. more than one order of magnitude smaller that was has been attained so far. Moreover, the improvement on the sensitivity of the tensor-to-scalar ratio (of the order of $\simeq 0.001$ with a similar systematic error [70]), would provide a confirmation of the existence of the inflationary cosmological GW background, if any.

\section{Acknowledgements}

NC's work is supported by NSF grant PHY-1204371. We thank Vuk Mandic for useful conversations.

\section{References}

[1] Wu C, Mandic V and Regimbau T 2012 Phys.Rev. D85 104024 (Preprint 1112.1898)

[2] Wu C J, Mandic V and Regimbau T 2013 Phys.Rev. D87 042002

[3] Marassi S, Schneider R and Ferrari V 2009 Mon.Not.Roy.Astron.Soc. 398293 (Preprint 0906. 0461)

[4] Grishchuk L 1975 Sov. Phys. JETP 40 409-415

[5] Starobinski A 1979 JETP Lett. 30 682-686

[6] Bar-Kana R 1994 Phys.Rev. D50 1157-1160 (Preprint astro-ph/9401050)

[7] Easther R, Giblin John T J and Lim E A 2007 Phys.Rev.Lett. 99221301 (Preprint astro-ph/ 0612294)

[8] Lopez A and Freese K 2013 (Preprint 1305.5855)

[9] Apreda R, Maggiore M, Nicolis A and Riotto A 2002 Nucl. Phys. B631 342-368 (Preprint gr-qc/0107033)

[10] Leitao L, Megevand A and Sanchez A D 2012 JCAP 1210024 (Preprint 1205.3070) 
Improved constraint on the primordial gravitational-wave density using recent cosmological data and its impa

[11] Kibble T W B 1976 J.Phys.A A 9 1387-1398

[12] Vilenkin A and Shellard E 1994 Cosmic Strings and Other Topological Defects (Cambridge University Press, Cambridge, England)

[13] Olmez S, Mandic V and Siemens X 2010 Phys.Rev. D81 104028 (Preprint 1004.0890)

[14] Brustein R, Gasperini M, Giovannini M and Veneziano G 1995 Phys. Lett. B361 45-51 (Preprint hep-th/9507017)

[15] Buonanno A, Maggiore M and Ungarelli C 1997 Phys. Rev. D55 3330-3336 (Preprint gr-qc/ 9605072)

[16] Aasi J et al. (LIGO Scientific Collaboration, VIRGO Collaboration) 2014 (Preprint 1406.4556)

[17] Shannon R, Ravi V, Coles W, Hobbs G, Keith M et al. 2013 Science 342 334-337 (Preprint 1310.4569)

[18] Kamionkowski M and Kosowsky A 1998 Phys.Rev. D57 685-691 (Preprint astro-ph/9705219)

[19] Turner M S 1997 Phys. Rev. D 55(2) R435-R439 URL http://link.aps.org/doi/10.1103/ PhysRevD.55.R435

[20] Allen B 1997 The Stochastic gravity wave background: Sources and detection (Preprint gr-qc/ 9604033)

[21] Maggiore M 2000 Phys.Rept. 331 283-367 (Preprint gr-qc/9909001)

[22] Cyburt R H, Fields B D, Olive K A and Skillman E 2005 Astropart.Phys. 23 313-323 (Preprint astro-ph/0408033)

[23] Smith T L, Pierpaoli E and Kamionkowski M 2006 Phys.Rev.Lett. 97021301 (Preprint astro-ph/ 0603144)

[24] Sendra I and Smith T L 2012 Phys.Rev. D85 123002 (Preprint 1203.4232)

[25] Ade P et al. (Planck Collaboration) 2013 (Preprint 1303.5075)

[26] Ade P et al. (Planck Collaboration) 2013 (Preprint 1303.5077)

[27] Anderson L, Aubourg E, Bailey S, Beutler F, Bolton A S et al. 2013 (Preprint 1303.4666)

[28] Mangano G, Miele G, Pastor S, Pinto T, Pisanti O et al. 2005 Nucl.Phys. B729 221-234 (Preprint hep-ph/0506164)

[29] Beringer J et al. (Particle Data Group) 2012 Phys.Rev. D86 010001

[30] Melchiorri A, Mena O and Slosar A 2007 Phys.Rev. D76 041303 (Preprint 0705.2695)

[31] Hannestad S, Mirizzi A, Raffelt G G and Wong Y Y 2010 JCAP 1008001 (Preprint 1004.0695)

[32] Fischler W and Meyers J 2011 Phys.Rev. D83 063520 (Preprint 1011.3501)

[33] Binetruy P, Deffayet C, Ellwanger U and Langlois D 2000 Phys.Lett. B477 285-291 (Preprint hep-th/9910219)

[34] Shiromizu T, Maeda K i and Sasaki M 2000 Phys.Rev. D62 024012 (Preprint gr-qc/9910076)

[35] Flambaum V and Shuryak E 2006 Europhys.Lett. 74 813-816 (Preprint hep-th/0512038)

[36] Calabrese E, Huterer D, Linder E V, Melchiorri A and Pagano L 2011 Phys.Rev. D83 123504 (Preprint 1103.4132)

[37] Blennow M, Fernandez-Martinez E, Mena O, Redondo J and Serra P 2012 JCAP 1207022 (Preprint 1203.5803)

[38] Caramete A and Popa L 2014 JCAP 1402012 (Preprint 1311.3856)

[39] Fixsen D 2009 Astrophys.J. 707 916-920 (Preprint 0911.1955)

[40] Reichardt C L, de Putter R, Zahn O and Hou Z 2012 Astrophys.J. 749 L9 (Preprint 1110.5328)

[41] Das S, Louis T, Nolta M R, Addison G E, Battistelli E S et al. 2013 (Preprint 1301.1037)

[42] Beutler F, Blake C, Colless M, Jones D H, Staveley-Smith L et al. 2011 Mon.Not.Roy.Astron.Soc. 416 3017-3032 (Preprint 1106.3366)

[43] Padmanabhan N, Xu X, Eisenstein D J, Scalzo R, Cuesta A J et al. 2012 Mon.Not.Roy.Astron.Soc. 427 2132-2145 (Preprint 1202.0090)

[44] Anderson L, Aubourg E, Bailey S, Bizyaev D, Blanton M et al. 2013 Mon.Not.Roy.Astron.Soc. 427 3435-3467 (Preprint 1203.6594)

[45] Ade P et al. (Planck Collaboration) 2014 Astron.Astrophys. 566 A54 (Preprint 1311.1657)

[46] James F and Roos M 1975 Comput. Phys. Commun. 10 343-367. 38 p 
[47] Blas D, Lesgourgues J and Tram T 2011 JCAP 1107034 (Preprint 1104.2933)

[48] Feldman G J and Cousins R D 1998 Phys.Rev. D57 3873-3889 (Preprint physics/9711021)

[49] Ade P et al. (Planck Collaboration) 2013 (Preprint 1303.5076)

[50] Forero D, Tortola M and Valle J 2012 Phys. Rev. D86 073012 (Preprint 1205.4018)

[51] Ade P et al. (Planck Collaboration) 2013 (Preprint 1303.5085)

[52] Jackson M G, Jones N T and Polchinski J 2005 JHEP 0510013 (Preprint hep-th/0405229)

[53] Damour T and Vilenkin A 2000 Phys.Rev.Lett. 85 3761-3764 (Preprint gr-qc/0004075)

[54] Bennett D P and Bouchet F R 1988 Phys.Rev.Lett. 60257

[55] Ringeval C, Sakellariadou M and Bouchet F 2007 JCAP 0702023 (Preprint astro-ph/0511646)

[56] Blanco-Pillado J J, Olum K D and Shlaer B 2014 Phys.Rev. D89 023512 (Preprint 1309.6637)

[57] Aasi J, Abadie J, Abbott B, Abbott R, Abbott T et al. 2014 Phys.Rev.Lett. 112131101 (Preprint 1310.2384)

[58] Siemens X, Creighton J, Maor I, Ray Majumder S, Cannon K et al. 2006 Phys.Rev. D73 105001 (Preprint gr-qc/0603115)

[59] Abbott B et al. (LIGO Scientific Collaboration, VIRGO Collaboration) 2009 Nature 460990 (Preprint 0910.5772)

[60] Ade P et al. (BICEP2 Collaboration) 2014 Phys.Rev.Lett. 112241101 (Preprint 1403.3985)

[61] Ade $\mathrm{P}$ et al. (The POLARBEAR Collaboration) 2014 (Preprint 1403.2369)

[62] Ahmed Z et al. (BICEP3 Collaboration) 2014 (Preprint 1407.5928)

[63] Austermann J, Aird K, Beall J, Becker D, Bender A et al. 2012 SPTpol: an instrument for CMB polarization measurements with the South Pole Telescope vol 8452 p 84520 E (Preprint 1210.4970)

[64] Niemack M, Ade P, Aguirre J, Barrientos F, Beall J et al. 2010 ACTPol: A polarization-sensitive receiver for the Atacama Cosmology Telescope vol 7741 p 77411S (Preprint 1006.5049)

[65] Reichborn-Kjennerud B, Aboobaker A M, Ade P, Aubin F, Baccigalupi C et al. 2010 EBEX: A balloon-borne CMB polarization experiment (Preprint 1007.3672)

[66] Filippini J P et al. 2010 SPIDER: a balloon-borne CMB polarimeter for large angular scales Society of Photo-Optical Instrumentation Engineers (SPIE) Conference Series (Society of Photo-Optical Instrumentation Engineers (SPIE) Conference Series vol 7741) (Preprint 1106.2158)

[67] Amendola L et al. (Euclid Theory Working Group) 2013 Living Rev.Rel. 166 (Preprint 1206.1225)

[68] Kitching T, Heavens A, Verde L, Serra P and Melchiorri A 2008 Phys.Rev. D77 103008 (Preprint 0801.4565)

[69] Abazajian K, Arnold K, Austermann J, Benson B, Bischoff C et al. 2013 (Preprint 1309.5383)

[70] Abazajian K, Arnold K, Austermann J, Benson B, Bischoff C et al. 2013 (Preprint 1309.5381) 